\title{
Nanobubbles Containing sPD-I and Ce6 Mediate Combination Immunotherapy and Suppress Hepatocellular Carcinoma in Mice
}

\author{
Yandi Tan $\mathbb{D}^{1, *}$ \\ Shiqi Yang (iD) ${ }^{1, *}$ \\ Yao $\mathrm{Ma}^{2}$ \\ Jinlin $\mathrm{Li}^{1}$ \\ Qian Xie \\ Chaoqi Liu' \\ Yun Zhao iD ' \\ 'Medical College of China Three Gorges \\ University, Yichang, Hubei, People's \\ Republic of China; ${ }^{2}$ Department of \\ Ultrasonography, Yichang Central \\ People's Hospital, Yichang, People's \\ Republic of China \\ *These authors contributed equally to \\ this work
}

Correspondence: Yun Zhao; Chaoqi Liu Medical College of China Three Gorges University, Yichang, Hubei, 443000,

People's Republic of China

Email zhaoyun@ctgu.edu.cn;

ctgulcq@163.com
Purpose: Immune checkpoint inhibitors (ICIs) and sonodynamic therapy (SDT) are types of immunotherapy. In order to combine soluble programmed cell death protein 1 (sPD-1)mediated immune checkpoint therapy and chlorin e6 (Ce6)-assisted SDT, nanobubbles (NBs) were generated to simultaneously load sPD-1 and Ce6.

Materials and Methods: The sPD-1/Ce6-NBs, which were prepared by thin-film hydration and mechanical oscillation, had a stable physical condition, and delivered sPD-1 and Ce6 in a targeted manner. NBs could strengthen tumor suppression by increasing tumor-targeting accumulation of Ce6 and sPD-1, and by inducing ultrasound-targeted NB destruction. A mouse H22 cell hepatoma xenograft model was used to evaluate the synergetic immunotherapeutic effect and mechanism of sPD-1/Ce6-NBs.

Results: By observing the tumor inhibition rate, tissue and cell apoptosis, apoptosis-related genes and protein expression, the best immunotherapeutic effect was exhibited by the sPD-1/ Ce6-NBs group. The immunotherapeutic mechanism initially demonstrated that when tumor cells were transfected by sPD-1 delivered by NBs, which downregulated the expression of programmed death-ligand 1 (PD-L1) in tumor cells, and blocked the PD-1/PD-L1 signaling pathway, which improved T-cell-mediated tumor inhibition. Furthermore, ICIs combined with SDT induced immunogenic cell death by translocating calreticulin to the cell surface and then synergistically enhancing antitumor immune responses.

Conclusion: In conclusion, sPD-1/Ce6-NBs were successfully designed. Ultrasoundmediated sPD-1/Ce6-NBs are potentially effective delivery systems for combination immunotherapy of hepatocellular carcinoma.

Keywords: immune checkpoint inhibitors, ultrasonics, drug delivery, tumor therapy, nanomaterials

\section{Introduction}

Hepatocellular carcinoma (HCC) is the fourth leading cause of cancer-associated mortality and the sixth most common tumor globally. ${ }^{1}$ The majority of patients with HCC are diagnosed at an advanced stage, and $70 \%$ of them have a recurrence within 5 years, can only be provided palliative treatment. Therefore, novel and effective therapies for HCC are urgently needed.

Recently, immune checkpoint inhibitors (ICIs) undergoing Phase III clinical trials have reshaped the treatment of advanced-stage HCC. ${ }^{2,3}$ Soluble programmed cell death 1 (sPD-1), the extracellular domain of PD-1, can be used as an ICI to block the PD-1/programmed death-ligand 1 (PD-L1) signaling pathway, which is 
one of the mechanisms by which tumor cells escape clearance by the host immune system. ${ }^{4}$ PD- 1 , which is expressed on the surface of T cells, is an important immunosuppressive regulatory molecule that can interact with PD-L1, which is expressed in the majority of tumor tissues. This interaction can activate tumor antigen specific $\mathrm{T}$ cells, and induces the immune tolerance of $\mathrm{T}$ cells, thus enhancing $\mathrm{T}$ cell-mediated antitumor immunity. The present study used sPD-1 delivered by nanobubbles (NBs) to transfect tumor cells that expressed SPD-1 in order to enhance the binding activity of PD-L1, and in turn block the PD-1/PD-L1 signaling pathway. However, neither first nor second-line randomized trials of ICIs have demonstrated a statistically significant improvement in overall survival. To achieve a more favorable synergistic therapy efficacy, ICIs combined with other treatments have been widely studied. ${ }^{5}$

Sonodynamic therapy (SDT) has gained considerable attention in cancer therapy. The basic principle of SDT is similar to that of photodynamic therapy (PDT), but the penetration depth of ultrasound in SDT can reach $5 \mathrm{~cm}$, which is more than that of PDT, and makes it possible to treat non-superficial tumors such as HCC. ${ }^{6,7}$ SDT can treat tumors directly and indirectly. To inhibit tumors directly, low-intensity ultrasound irradiation activates sonosensitizers, which then generate reactive oxygen species (ROS). ${ }^{8,9}$ ROS induce local cytotoxicity or damage the tumor-associated vasculature. To further inhibit tumors indirectly, ROS can generate immunogenic cell death (ICD) and then release tumor-associated antigens, which are recognized by dendritic cells (DCs) to activate immune cells, thus enhancing antitumor immune responses, including innate and adaptive immune responses. ${ }^{10}$ The present study used chlorin e6 (Ce6) as a sonosensitizer, which has shown improved pharmacokinetics, less toxicity and higher tumor affinity than those of other sonosensitizers. ${ }^{11}$ Furthermore $\mathrm{Ce} 6$, as an endogenous fluorochrome, could be irradiated to emit light. Thus, Ce6 was used to analyze the pharmacokinetics of sPD-1/ Ce6-NBs.

NBs are small gas-filled bubbles with diameters of $<1$ $\mu \mathrm{m}$. NBs have various advantages, including low cost, non-invasiveness, real-time imaging, avoidance of hazardous ionizing radiation, and a favorable balance between sensitivity and resolution. NBs are both contrast-imaging agents and drug-delivery systems, and therefore capable of achieving a non-invasive combination of diagnostics and therapeutics. ${ }^{12}$ As contrast-imaging agents, in addition to anatomic imaging, NBs are used in imaging phenotypes at a cellular level, contributing to disease detection and assessment. $^{13}$ As delivery systems, NBs, by delivering chemical agents, genes and protein materials in a targeted manner, ${ }^{14,15}$ enhance the treatment effect and prevent side effects. Their nanometer size facilitates their movement through the capillary endothelial cell gap to the tumor, thus causing enhanced permeability and retention (EPR) effect. The accumulation of NBs is increased in tumor tissue and decreased in healthy tissue. Furthermore, ultrasound-targeted NB destruction (UTND), causing NBs to oscillate and rupture under local ultrasound stimulation, changes the integrity and permeability of the vessel wall and cell membrane, ${ }^{16}$ thus allowing more drug to enter into targeted cells. ${ }^{17,18}$ UTND can also produce the cavitation and sonoporation effect, boosting therapeutic efficiency. In our study, NBs could prolong the half-life of Ce6 in vivo and resulted in better gene transfection efficiency and biosafety of sPD-1 than those of viral vectors.

In the present study, sPD-1 and Ce6 co-loading NBs were constructed with the assistance of UTND, and SPD-1 and $\mathrm{Ce} 6$ were targeted released and accumulated in tumors, where sPD-1 acted as ICI and Ce6 was activated to induce SDT, jointly achieving an enhanced cancer immunotherapy effect.

\section{Materials and Methods Preparation of sPD- I/Ce6-NBs}

sPD-1/Ce6-NBs were prepared using the thin-film hydration method. ${ }^{19}$ Briefly, 1,2-dipalmitoyl-sn-glycero-3-phosphocholine (DPPC): Polyethylene glycol 2000 (PEG2000): $\quad 3$ '[N- (N', N'-dimethylaminoethane) carbamoyl]cholesterol (DC-Chol):Ce6 (Sigma-Aldrich; Merck $\mathrm{KGaA}$ ) at a mass ratio of 5:2:2:0.5 were added to chloroform in a glass vial. Chloroform was then removed using a rotary evaporator (Precision HLG3; Heidolph), which formed a homogeneous lipid film. Next, a mixture of PBS and glycerin at a volume ratio of 9:1 was added to dissolve the film. The mixture was divided into sealed glass vials. The air in the vials was replaced by perfluoropropane gas (Wuhan Newradar Special Gas Co., Ltd., Wuhan, China) using a self-made gas exchange device. The glass vial was mechanically shaken for $60 \mathrm{sec}$ in a silver-mercury mixer (SI Vortex-Genie 2; Scientific Industries; Thermo Fisher Scientific, Inc.), and then incubated with sPD-1 plasmid for $20 \mathrm{~min}$ at room temperature. 


\section{Characterization of sPD-1/Ce6-NBs}

Morphological characterization was performed with a scanning electron microscopy (SEM; JSM-7500F; JEOL, Ltd.). The mean diameter, size distribution and electric potential were obtained with Zetasizer Nano ZS (Malvern Panalytical). The UV-vis absorption spectrum of Ce6-NBs was determined with a Microplate Reader (SpectraMax 190, Molecular Devices, Wuhan, China). The sPD-1 loading capacity was verified by laser scanning confocal microscopy (LSCM; Nikon Corporation, Tokyo, Japan) and gel electrophoresis. Briefly, the SPD-1 plasmid was incubated with phycoerythrin (AAT Bioquest, US) for $30 \mathrm{~min}$ at room temperature and then used to construct SPD-1-NBs, which were observed under a laser scanning confocal microscope. The sPD-1 plasmid in the NBs was quantified by $1 \%$ agarose. In total, $1 \mu \mathrm{g} \mathrm{sPD}-1$ plasmid was incubated with different volumes of NBs $(4,8,12,16$ or $20 \mu \mathrm{L})$ for $20 \mathrm{~min}$ at room temperature and then subjected to electrophoresis (110 V, $40 \mathrm{~min}$ ). The negatively charged plasmid DNA combined with cationic NBs showed no band, while the unbound free sPD-1 plasmid migrated to the anode and thus appeared as a bright band on the gel. Thus, when no band was observed, the volume of NBs was optimal to load $1 \mu \mathrm{g}$ sPD-1. Therefore, the quantity of sPD-1 plasmid in NBs could be quantified by calculating the volume of NBs.

The loading capacity of $\mathrm{Ce} 6$ in sPD-1/Ce6-NBs was observed by laser scanning confocal microscopy. The UVvis absorption spectra of Ce6-NBs and Ce6 were obtained by UV spectrophotometry. The Ce6 encapsulation efficiency was calculated as follows: The maximum absorption, standard curve and recovery rate were obtained by UV spectrophotometry. The Ce6-NBs were dialyzed using a dialysis bag (Union Carbide, US) for $6 \mathrm{~h}$ to filter the free $\mathrm{Ce} 6$ and then mixed with methyl alcohol to resolve NBs. ${ }^{20}$ Now the mixture was the Ce6 all loaded into NBs. Next, the OD of the mixture was measured at its maximum absorption, and the quantity of $\mathrm{Ce} 6$ was calculated according to the standard curve. The encapsulation efficiency and Loading capacity was calculated with the following equation: Encapsulation efficiency (\%) $=$ (Quantity of Ce6 loaded onto NBs/total quantity of Ce6 material) x $100 \%$. Loading capacity $(\%)=($ Quantity of Ce6 loaded onto NBs/total quantity of Ce6-NBs) x $100 \%$.

\section{Animal Model Establishment}

Male BALB/c mice (18-20 g) were supplied by Wuhan Institute of Biological Products Co., Ltd. (Wuhan, China).
All animal experiments were approved by the Animal Experimental Committee of China Three Gorges University (Hubei, China). All procedures were performed following the guidelines of the Ministry of Science and Technology of the People's Republic of China. For developing HCC, H22 cells were purchased from China Center for Type Culture Collection (CCTCC, Wuhan, China), and cultured in RPMI 1640 (Thermo Fisher, Waltham, MA, USA) supplemented with 10\% FBS (Thermo Fisher) and $1 \%$ penicillin/streptomycin (Thermo Fisher) at $37^{\circ} \mathrm{C}, 5 \%$ $\mathrm{CO}^{2}$. Then mice were subcutaneously injected with $1 \times 10^{7}$ $\mathrm{H} 22$ cells into the right axilla. The tumor volume (V) was determined using the following equation: $\mathrm{V}=\left(\mathrm{A} \times \mathrm{B}^{2}\right) / 2$, where $\mathrm{A}$ is the tumor length and $\mathrm{B}$ the tumor width. When tumors reached $\sim 100 \mathrm{~mm}^{3}$, in vivo experiments were carried out.

\section{In vivo Fluorescence Imaging}

The tumor-targeting ability and histological distribution of sPD-1/Ce6-NBs were evaluated with the IVIS Spectrum in vivo imaging system for small animals (Xenogen, US). Free Ce6 and sPD-1/Ce6-NBs, which had the same quantity of $\mathrm{Ce} 6$, were intravenously injected into the mice, and the mice were anaesthetized prior to imaging with isoflurane gas at an induction dose of $2-3 \%$ and maintenance dose of $1.5-2 \%$. Next, fluorescence (FL) images were acquired with the IVIS Spectrum in vivo imaging system (excitation wavelength, $620 \mathrm{~nm}$; emission wavelength, 670 $\mathrm{nm})$ at different time points post-injection $(0,0.5,1,4,6$, 8,10 and $24 \mathrm{~h}$ ). At $24 \mathrm{~h}$ post-injection, animals were humanely sacrificed by cervical dislocation. And the tumors and main organs, including the heart, liver, spleen, lung and kidneys, were collected for FL imaging. Frozen sections of the tumor were prepared to observe the Ce6 fluorescence intensity by fluorescence microscopy (Ti-DH; Nikon Corporation). Semi-quantification was conducted with Image $\mathrm{J}$ software 1.51k (ACEA Bioscience, Inc.; Agilent Technologies, Inc.).

\section{In vivo Antitumor Efficacy}

To evaluate the antitumor efficacy of sPD-1/Ce6-NBs in vivo, 35 mice bearing subcutaneous $\mathrm{H} 22$ tumors were randomly divided into the following six groups: i) Control; ii) empty NBs; iii) Ce6; iv) SPD-1-NBs; v) Ce6NBs; and vi) SPD-1/Ce6-NBs. Each mouse received the same volume of NBs or PBS through tail vein injection every 3 days (five times). The control group received PBS. The Ce6 group received a mixture of Ce6 and 
PBS. Other groups received the corresponding NBs. The same volume of Ce6, Ce6-NBs and SPD-1/Ce6-NBs had the same mass of Ce6. With the exception of the control group, the treatment groups were exposed to ultrasound irradiation (UGT1025; Chongqing Medical University) with the following parameters: Frequency, $1.1 \mathrm{MHz}$; intensity, $1.8 \mathrm{~W} / \mathrm{cm}^{2}$; time, $2 \mathrm{~min}$; and duty cycle, $50 \%$. Tumors of the Ce6, Ce6-NBs and sPD-1/Ce6-NBs groups were exposed to ultrasound again to induce SDT at 6 $\mathrm{h}$ post-injection. The longest (L) and shortest (S) diameters of the tumors were measured before each treatment. The tumor volume was calculated to obtain the tumor growth curve. The body weight of the mice was determined to calculate the weight curve. On the 16th day, the mice were euthanized by cervical dislocation, and the tumors and spleen were weighed and collected. The volume and weight inhibition rates were calculated. The mice and their livers were weighed to obtain the liver index, which can reveal the toxicity of sPD-1/Ce6-NBs. Parts of tumor samples were rapidly frozen, while other parts were treated with formalin for 1 day and then paraffin embedded. To evaluate the histopathological changes, the paraffin-embedded tissue was cut into sections of $4 \mu \mathrm{m}$ in thickness, and then stained with hematoxylin and eosin (H\&E) before being observed under light microscopy (Nikon Corporation).

\section{Reverse Transcription-Quantitative PCR (RT-qPCR)}

To evaluate the antitumor efficacy and mechanism at the genetic level, the relative mRNA expression levels of B-cell lymphoma-2-associated X (Bax), B-cell lymphoma-2 (Bcl-2), cluster of differentiation 80 (CD80), CD86, tumor necrosis factor alpha (TNF- $\alpha)$, interferon gamma (IFN- $\gamma$ ), interleukin-2 (IL-2) and PD-L1 were determined by RT-qPCR. Total miRNA from tumors was extracted using the mirVana miRNA Isolation kit (Ambion; Thermo Fisher Scientific, Inc.); subsequently reverse transcribed to cDNA using the Universal cDNA Synthesis Kit (Exiqon; Qiagen, Inc.); and finally analyzed using a CFX96 Touch Real-Time PCR System (Bio-Rad Laboratories, Inc.). The thermocycling parameters were $95^{\circ} \mathrm{C}$ for $30 \mathrm{sec}$, followed by 40 cycles of $95^{\circ} \mathrm{C}$ for $5 \mathrm{sec}$ and $60^{\circ} \mathrm{C}$ for $30 \mathrm{sec}$. GAPDH served as a control, and the relative expression levels of Bax, Bcl-2, CD80, CD86, INF- $\gamma$, TNF- $\alpha$, IL-2 and PD-L1 were evaluated by the quantification cycle method. The primers used in RTqPCR are shown in Table 1.

\section{Analysis of Tumor Cell Apoptosis}

For assessing the apoptosis of the tumors, the paraffinembedded sections were stained with a TUNEL Assay kit (Hoffman-LaRoche Ltd, Basel, Switzerland) according to the manufacturer's instructions and observed by fluorescence microscopy (Nikon Corporation). Semiquantification was carried out by calculating fluorescence intensity with Image J $1.51 \mathrm{k}$ software (National Institutes of Health).

\section{Analysis of Bax, Bcl-2, Calreticulin (CRT) and PD-LI Protein}

To evaluate the antitumor efficacy and mechanism at the protein level, the protein expression levels of Bcl-2, Bax, CRT and PD-L1 were evaluated by immunohistochemistry. After antigen retrieval, the paraffin-embedded sections were incubated with primary antibodies against Bax, Bcl2, CRT or PD-L1 overnight at $4^{\circ} \mathrm{C}(1: 200$ dilution in $1 \%$ Bovine albumin (R\&D System, Minneapolis, MN, USA); Santa Cruz Biotechnology, Inc.) and then incubated with goat anti-rabbit IgG (Jackson ImmunoResearch Inc., West Grove, PA, USA). After being stained with 4',6-diamidino2-phenylindole (DAPI) (Sigma) for PD-L1 or DAB (Biological Technology co. ltd) for others, all sections were observed under a fluorescence microscope and quantified using Image J software $1.51 \mathrm{k}$.

\section{Analysis of Cytotoxic T Cell (CTL)-Specific and Natural Killer (NK) Cell Responses}

To explore whether sPD-1/Ce6-NBs caused a synergetic immunotherapy effect, CTL-specific and NK cell responses were evaluated as follows: For NK cell activity analysis, under aseptic conditions, the mouse spleens were pulverized, filtered and washed to obtain splenic cells. At a ratio of effector cells (splenic cells): target cells (H22 cells) of 100:1, 50:1 and 25:1, splenic cells were cultured with H22 cells in 96-well plates for $3 \mathrm{~h}$. Finally, NK cell activity was measured with the lactate dehydrogenase Assay kit (Beyotime Institute of Biotechnology).

For CTL-specific activity analysis, in contrast to the aforementioned NK experiment, the prepared splenic cells were stimulated by $\mathrm{H} 22$ antigen (tumor cell lysis) for 48 h. Next, activated lymphocytes were mixed with $\mathrm{H} 22$ cells 
Table I Primers for PCR Reaction

\begin{tabular}{|c|c|c|}
\hline Genes & Primer & Sequences of Primer ( $\left(5^{\prime}\right.$ to $\left.3^{\prime}\right)$ \\
\hline GAPDH & $\begin{array}{l}\text { Forward } \\
\text { Reverse }\end{array}$ & $\begin{array}{l}\text { GGTGGTCTCCTGTGACTTCAA } \\
\text { CCACCCTGTTGCTGTAGCC }\end{array}$ \\
\hline BCl-2 & $\begin{array}{l}\text { Forward } \\
\text { Reverse }\end{array}$ & $\begin{array}{l}\text { CGTCAACAGGGAGATGTCACC } \\
\text { CAGCCAGGAGAAATCAAACAGAG }\end{array}$ \\
\hline Bax & $\begin{array}{l}\text { Forward } \\
\text { Reverse }\end{array}$ & $\begin{array}{l}\text { TGGTTGCCCTCTTCTACTTTGC } \\
\text { CAGACAAGCAGCCGCTCAC }\end{array}$ \\
\hline$I N F-\gamma$ & $\begin{array}{l}\text { Forward } \\
\text { Reverse }\end{array}$ & $\begin{array}{l}\text { CCATCGGCTGACCTAGAGAA } \\
\text { GATGCAGTGTGTAGCGTTCA }\end{array}$ \\
\hline$T N F-\alpha$ & $\begin{array}{l}\text { Forward } \\
\text { Reverse }\end{array}$ & $\begin{array}{l}\text { TCAACCTCCTCTCTGCCGTC } \\
\text { GAGCAATGACTCCAAAGTAGACCTG }\end{array}$ \\
\hline CD86 & $\begin{array}{l}\text { Forward } \\
\text { Reverse }\end{array}$ & $\begin{array}{l}\text { GGCAAGGCAGCAATACCTTA } \\
\text { CTCTTTGTGCTGCTGATTCG }\end{array}$ \\
\hline CD80 & $\begin{array}{l}\text { Forward } \\
\text { Reverse }\end{array}$ & $\begin{array}{l}\text { TCTCCACGGAAACAGCATCT } \\
\text { CTTACGGAAGCACCCATGAT }\end{array}$ \\
\hline IL-2 & $\begin{array}{l}\text { Forward } \\
\text { Reverse }\end{array}$ & $\begin{array}{l}\text { GCGAATTCATGATGTGCAAAGTACTG } \\
\text { GCGTCGACTTATTTTTGCAGATATCTCAC }\end{array}$ \\
\hline PD-LI & $\begin{array}{l}\text { Forward } \\
\text { Reverse }\end{array}$ & $\begin{array}{l}\text { TGCTGCATAATCAGCTACGG } \\
\text { CCACGGAAATTCTCTGGTTG }\end{array}$ \\
\hline sPDI & $\begin{array}{l}\text { Forward } \\
\text { Reverse }\end{array}$ & $\begin{array}{l}\text { CATGAGATCTATGCAGATCCCACAGGCG } \\
\text { CATGCTCGAGTTAGAACTGGCCGGCTGGCCT }\end{array}$ \\
\hline
\end{tabular}

Abbreviations: GAPDH, glyceraldehyde-3-phosphate dehydrogenase; Bcl-2, B-cell lymphoma-2; Bax, B-cell lymphoma-2-associated $\mathrm{X}$; IFN- $\gamma$, interferon gamma; TNF- $\alpha$, tumor necrosis factor alpha; CD86, cluster of differentiation 86; CD80, cluster of differentiation 80; IL-2, interleukin-2; PD-LI, programmed death-ligand I; SPD-I, soluble programmed cell death I.

for $3 \mathrm{~h}$ at a ratio of 100:20. The percentage of $\mathrm{LDH}$ cytotoxicity was determined as follows: LDH cytotoxicity $(\%)=($ Experimental release - spontaneous release $) /(\max -$ imum release - spontaneous release) x 100\%.

\section{Statistical Analysis}

Statistical analysis was performed using SPSS software 19.0.0. Comparisons between two groups were performed with Student's $t$-test). $P<0.05$ was considered to indicate a statistically significant difference.

\section{Results}

\section{Characterization of sPD-1/Ce6-NBs}

sPD-1/Ce6-NBs were synthesized by thin-film hydration and mechanical oscillation, and the resulting suspension was dark green. As shown in Figure 1A, sPD-1/Ce6-NBs were composed of a lipid shell and a perfluoropropane gas core. Their hydrodynamic size was $282.97 \pm 21.80 \mathrm{~nm}$ with the dispersity index of 0.174 , indicating that NBs had a good dispersity (Figure 1B). NBs were spherical and were dispersed uniformly (Figure 1C) and their $\zeta$ potential was $16.50 \pm 0.79 \mathrm{mV}$ (Figure 1D). Red fluorescence of Ce6 and SPD-1 was exhibited on the surface of sPD-1-NBs and Ce6-NBs, respectively (Figure $1 \mathrm{E}$ and F). According to agarose, no band was observed when the NBs volume was $20 \mu \mathrm{L}$. Thus, the DNA loading capacity of $20 \mu \mathrm{L}$ NBs was $1 \mu \mathrm{g}$ plasmid (Figure $1 \mathrm{G}$ ).

The spectrum analysis showed that the maximum absorption wavelength of Ce6 was $403 \mathrm{~nm}$. The standard curve covered $0.5-16 \mu \mathrm{g} / \mathrm{mL}$ in terms of the concentration range. The linear correlation between concentration (A) and absorbance (C) was good, and the regression equations were: $\mathrm{A}=0.115 \times \mathrm{C}+0.2176(\mathrm{r}=0.9975$; $\mathrm{n}=6)$. After $6 \mathrm{~h}$ of dialysis, the recovery rate of free Ce6 was 98.53 $\pm 0.24 \%$. The encapsulation efficiency and loading capacity were $40.85 \pm 3.09 \%$ and $1.32 \pm 0.10 \%$ respectively. The UVvis absorption spectrum of Ce6-NBs was similar to that of free $\mathrm{Ce} 6$, implying that the chromophore of $\mathrm{Ce} 6$ did not 
A

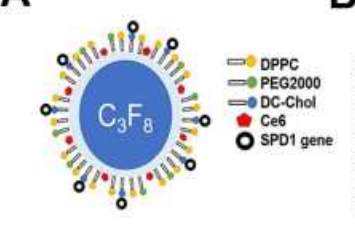

E

E

G

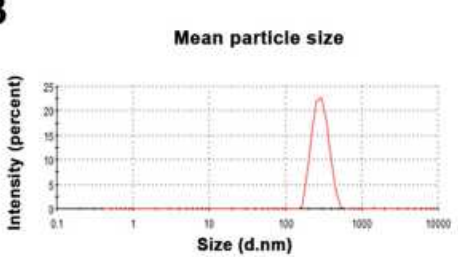

\section{C}

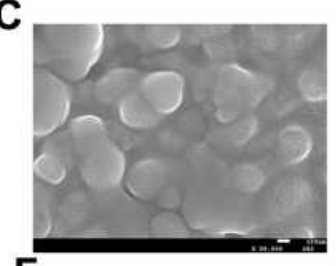

F

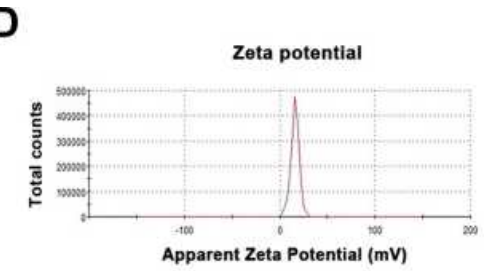

Apparent Zeta Potential (mV)

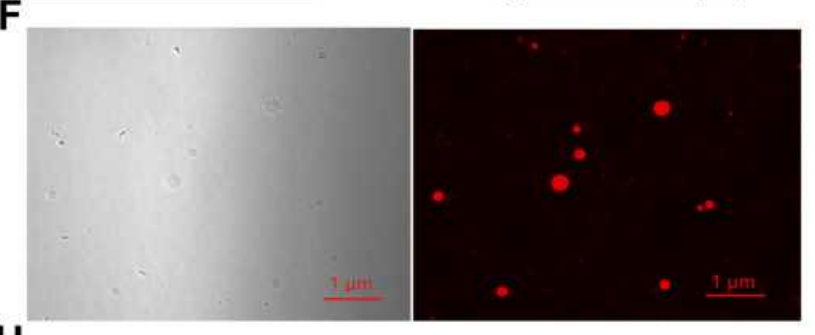

H

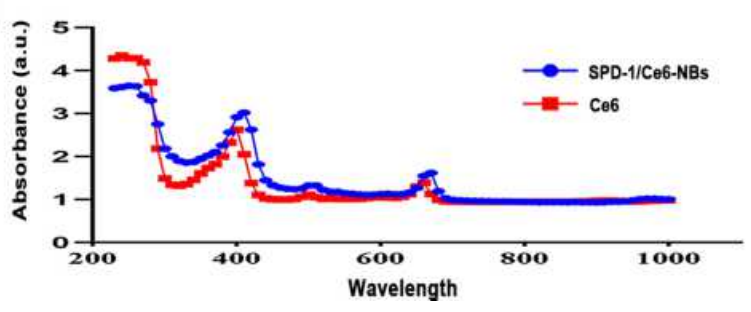

Figure I Characterization of sPD-I/Ce6-NBs. (A) Schematic diagram of sPD-I/Ce6-NBs. (B) Diameter and the mean particle size of sPD-I/Ce6-NBs. (C) Scanning electron microscopy of sPD-I/Ce6-NBs (magnification, x50,000). (D) Zeta potential of sPD-I/Ce6-NBs. (E) Red fluorescence of sPD-I on the surface of sPD-I-NBs under a laser scanning confocal microscope (magnification, xl0000). (F) Red fluorescence of Ce6 on the surface of sPD-I/Ce6-NBs under a fluorescence microscope (magnification, x200). (G) Gel electrophoresis showing the DNA loading capacity of sPD-I/Ce6-NBs. (H) UV-vis absorption spectra of sPD-I/Ce6-NBs and free Ce6, which showed no variation.

Abbreviations: C3F8, octafluoropropane; DPPC, I,2-dipalmitoyl-sn-glycero-3-phosphocholine; PEG2000, polyethylene glycol 2000; DC-Chol, 3'[N-(N', N'dimethylaminoethane)-carbamoyl]cholesterol; SPD-I, soluble programmed cell death I; Ce6, chlorin e6; NBs, nanobubbles.

vary upon encapsulation (Figure $1 \mathrm{H}$ ). Thus, NBs were efficient delivery systems for Ce6 and sPD-1.

\section{Pharmacokinetic Study of sPD-I/Ce6- NBs}

The present study evaluated the pharmacokinetics of sPD1/Ce6-NBs to verify whether NBs could prolong circulation time to achieve targeted delivery of $\mathrm{Ce} 6$ in vivo. FL imaging of mice was obtained with a small-animal imaging system. The fluorescence intensity in tumor tissues within $24 \mathrm{~h}$ demonstrated that $\mathrm{Ce} 6$ had a preferential localization in tumors, but a rapid clearance and low accumulation in healthy tissues. The difference was most evident at $6 \mathrm{~h}$. Thus, $6 \mathrm{~h}$ was selected as the optimum time of ultrasound exposure to induce SDT, with the best therapeutic efficacy in cancer and the lowest side effect in healthy tissues.

It was also demonstrated that NBs could increase the tumor accumulation of Ce6. The FL signal in the Ce6 tumor group gradually disappeared after $8 \mathrm{~h}$ of injection, while it was present in the sPD-1/Ce6-NBs group until 24 $\mathrm{h}$ post-injection (Figure 2A and B). Quantitative analysis showed that the mean FL intensity of the tumor in the sPD-1/Ce6-NBs group was 3.59-fold higher than that in the Ce6 group (Figure $2 \mathrm{C}$ and $\mathrm{D} ; P<0.01$ ). The results obtained in the frozen tissue sections were consistent with the results of FL imaging. The red fluorescence intensity in the sPD-1/Ce6-NBs tumors was higher than that in the $\mathrm{Ce} 6$ group, revealing that NBs could increase the accumulation of $\mathrm{Ce} 6$ in the tumor (Figure 2E and F; $P<0.01)$.

\section{Tumor Inhibition Rate After in vivo} Treatment

The antitumor effect was observed following in vivo treatment. A schematic illustration shows the treatment process (Figure $3 \mathrm{~A}$ ). The corresponding tumor growth curves (Figure 3B), and the tumor weight and volume inhibitory rates (Table 2) all showed the same trend of tumor suppression. In the control group, the tumor growth was rapid, with all treatments effectively delaying tumor growth in varying degrees (all $P<0.01$ ). The sPD-1/Ce6-NBs groups exhibited a marked therapeutic effect, and the tumor volume and weight inhibitory rates 

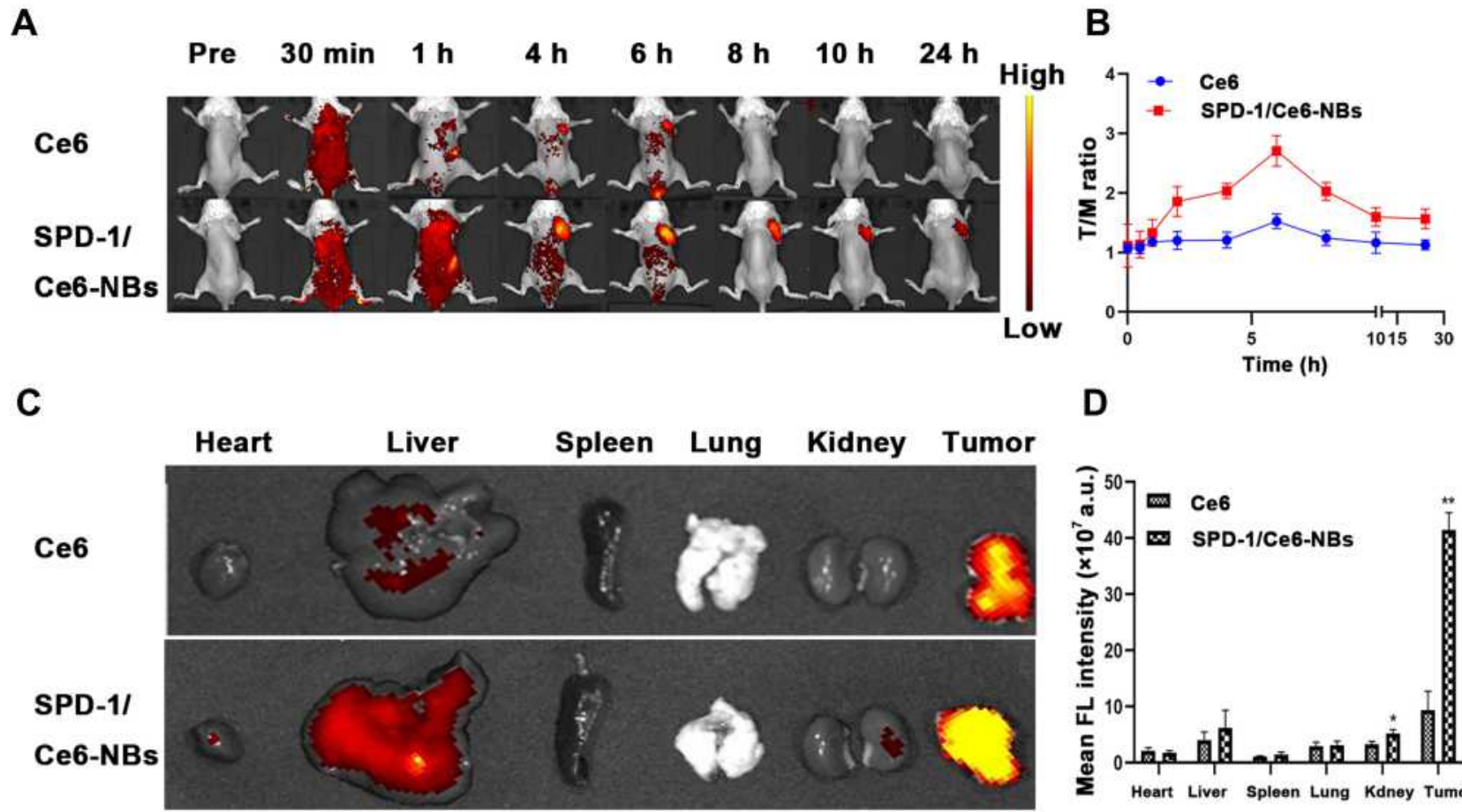

D

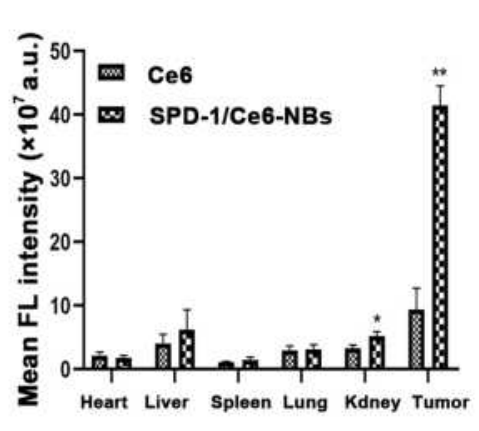

E

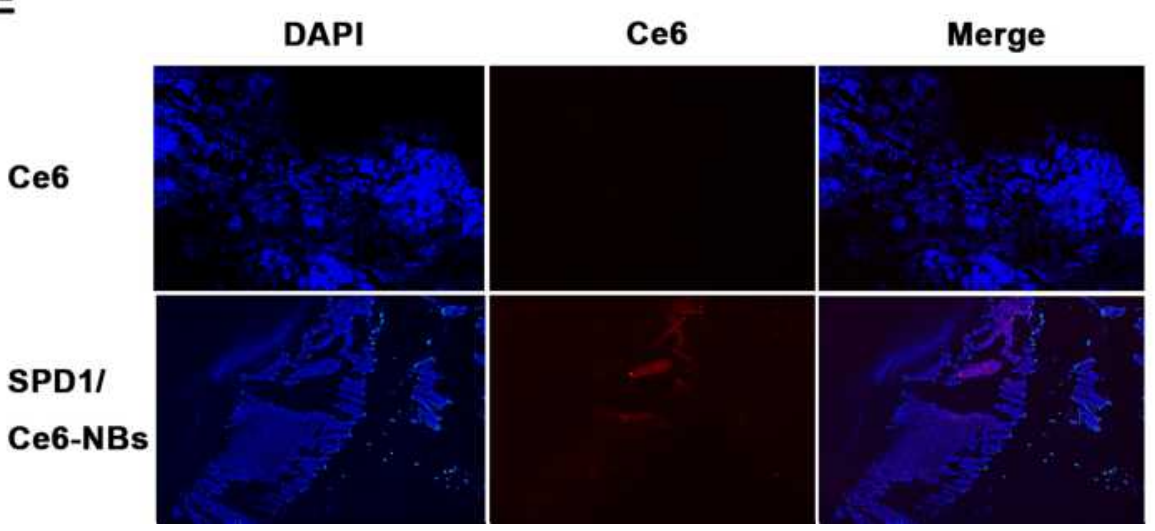

$\mathbf{F}$

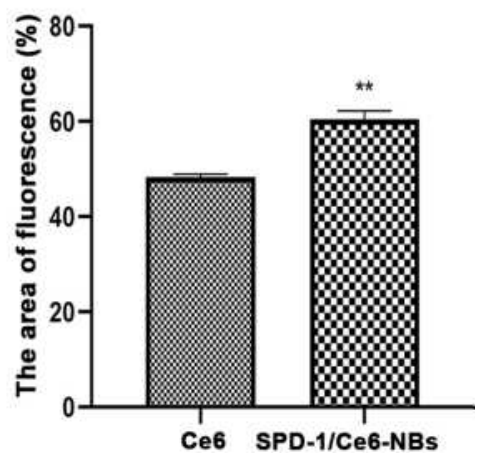

Figure 2 Pharmacokinetic study of sPD-I/Ce6-NBs. The tumor tissue of the sPD-I/Ce6-NBs group had more Ce6 accumulated in terms of time and concentration than that of the Ce6 group. (A) In vivo FL imaging of mice after intravenous injection of Ce6 and sPD-I/Ce6-NBs. (B) Semi-quantitative analysis of the FL signal of the tumor region in panel (A). (C) Ex vivo FL imaging of the heart, liver, spleen, lung, kidney and tumors at $24 \mathrm{~h}$ post-injection. (D) Quantitative analysis of the various organs shown in panel (C). (E) Images of frozen tumor sections at $24 \mathrm{~h}$ post-injection (magnification, $\mathrm{x} 400$ ). (F) Semi-quantification of the fluorescence intensity of Ce6 shown in panel. (*P $<0.05$, **P $<0.0$ l versus the $\mathrm{Ce} 6$ group).

Abbreviations: SPD-I, soluble programmed cell death I; Ce6, chlorin e6; NBs, nanobubbles; FL, fluorescence; DAPI, 4', 6-diamidino-2-phenylindole.

were $77.43 \pm 2.75$ and $82.51 \pm 1.19 \%$, respectively. Thus, SPD-1/Ce6-NBs exhibited a marked therapeutic effect. In addition, the weight of the mice did not decrease significantly during the treatment period (Figure 3C), and there was no significant difference in liver index between the groups (Figure 3D) (all $P>0.05$ ). The results of mice weight curve and liver index showed that NBs had no obvious toxicity towards mice. Furthermore, during the drug treatment period, no obvious adverse reaction was observed.

\section{Apoptosis of Tumor Cells}

To evaluate the antitumor activity, tumor histopathological changes were observed by H\&E staining, and cell apoptosis levels were determined by a TUNEL assay. In the treatment groups, particularly in the sPD-1/Ce6-NBs group, the results of $\mathrm{H} \& \mathrm{E}$ staining revealed severe tumor cell apoptosis or necrosis of the tumor cells (Figure 4A), while hardly any injuries was observed in the control group, which result is in agreement with that obtained by TUNEL assay (Figure 4B and C; $P<0.01$ ). 

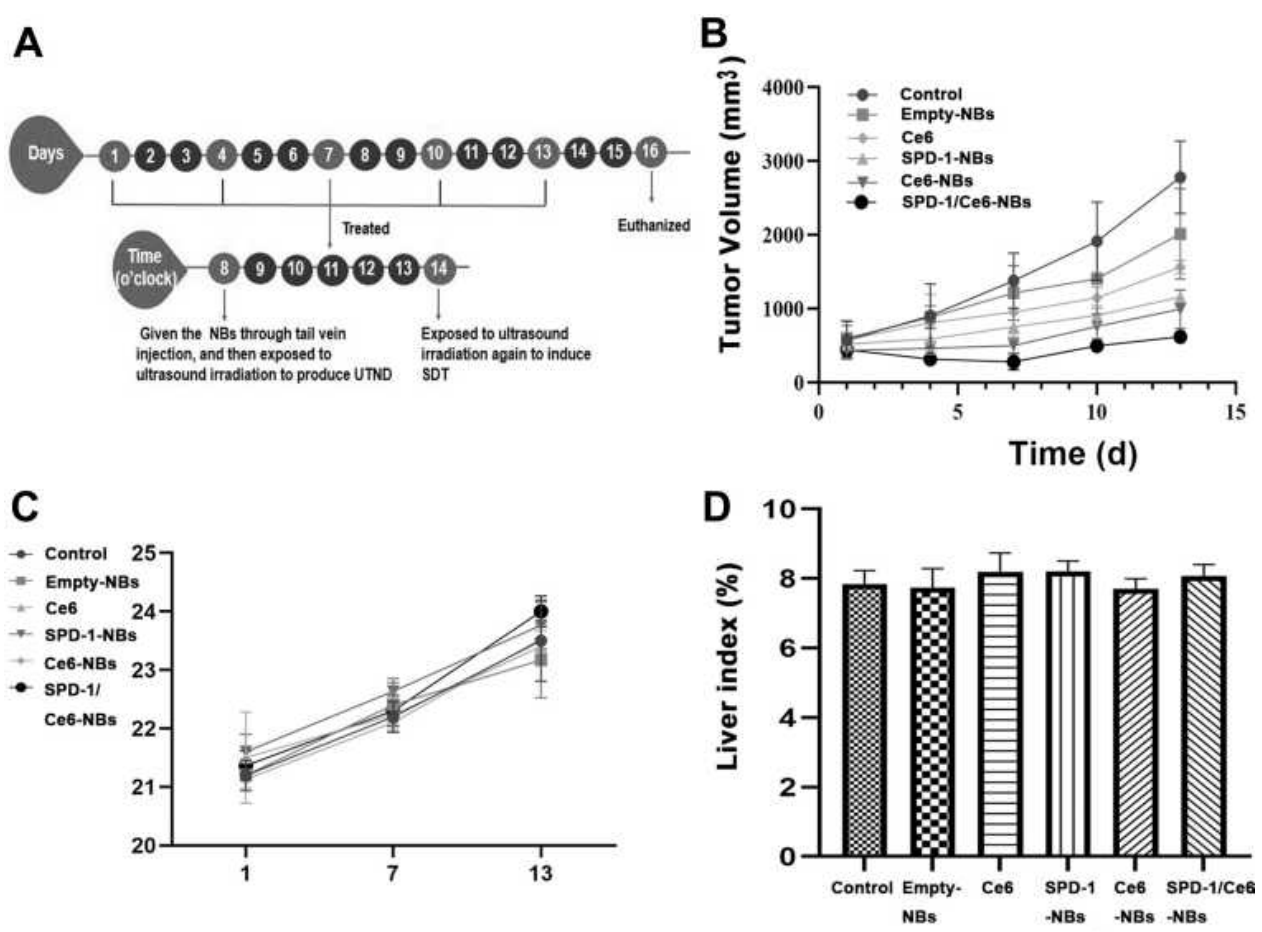

Figure 3 Tumor inhibition rate after in vivo treatment. (A) Schematic illustration showing the treatment process of sPD-1/Ce6-NBs. The same volume of NBs or PBS was administered to the animals through tail vein injection every 3 days (five times in total). With the exception of the control group, tumors were exposed to ultrasound irradiation immediately after injection, and the tumors of the Ce6, Ce6-NBs and SPD-I/Ce6-NBs groups were exposed to ultrasound irradiation again at $6 \mathrm{~h}$ post-injection. The curves of tumor growth shown in panel (B) indicated that all treatments effectively delayed tumor growth in varying degrees. The sPD-I/Ce6-NBs groups exhibited a marked therapeutic effect. The body weight of mice at different times shown in panel (C) and the liver index of mice in the different groups shown in panel (D) indicated that sPD-I/Ce6-NBs were no toxic in tumor.

Abbreviations: UTND, ultrasound-targeted NB destruction; SDT, sonodynamic therapy; SPD-I, soluble programmed cell death I; Ce6, chlorin e6; NBs, nanobubbles; d, day.

Furthermore, the expression of apoptosis-related genes and proteins was explored. Decreased Bcl-2 mRNA and protein expression, and enhanced Bax expression promote the apoptosis process. The results of RT-qPCR showed that, in all the treatment groups, the gene expression of Bax was higher than that in the control groups, with the sPD-1/Ce6-NBs group being the most obvious (Figure 4E). The opposite results were obtained for Bcl-2. Immunohistochemistry revealed the same tendency at the protein level (Figure 4D and F) (all $P<0.01$ ). Overall, these results, including tumor inhibition rate, histopathological changes, cell apoptosis and apoptosis-related expression, indicated that, compared with that of monotherapy, sPD-1/ Ce6-NBs-mediated combined immunotherapy could achieve a superior antitumor activity.

\section{Immune Activity is Enhanced by sPD-I/ Ce6-NBs in vivo}

To study the antitumor mechanism, first the present study verified the antitumor mechanism of sPD-1,

Table 2 The Tumor Volume and Weight on the 16th Day and Inhibition Rate

\begin{tabular}{|l|l|l|l|l|}
\hline Groups & Tumor Volume $\left(\mathbf{m m}^{\mathbf{3}}\right)$ & Tumor Weight $\mathbf{( g )}$ & Volume Inhibition Rate (\%) & Weight Inhibition Rate (\%) \\
\hline Control & $2780.60 \pm 489.87$ & $3.55 \pm 0.99$ & 0 & 0 \\
Empty-NBs & $1947.61 \pm 5 I 7.13$ & $2.49 \pm 0.55$ & $30.74 \pm 6.68^{* *}$ & $28.98 \pm 5.57^{* *}$ \\
Ce6 & $1632.68 \pm 131.25^{* *}$ & $1.7 I \pm 0.2 I^{*}$ & $40.57 \pm 6.07^{* *}$ & $44.08 \pm 7.78^{* *}$ \\
SPD-I-NBs & $1159.4 I \pm 5 I .69^{* *}$ & $1.64 \pm 0.06^{*}$ & $57.60 \pm 6.03^{* *}$ & $47.67 \pm 5.26^{* *}$ \\
Ce6-NBs & $996.58 \pm 260.35^{*}$ & $0.89 \pm 0.29^{*}$ & $64.39 \pm 4.13^{* *}$ & $74.38 \pm 6.2^{* *}$ \\
SPD-I/Ce6-NBs & $620.17 \pm 66.26^{* *}$ & $0.58 \pm 0.07^{*}$ & $77.43 \pm 2.75^{* *}$ & $82.5 I \pm 1.19 * *$ \\
\hline
\end{tabular}

Note: $* \mathrm{P}<0.05, * * \mathrm{P}<0.01$ versus the control group.

Abbreviations: SPD-I, soluble programmed cell death I; Ce6, chlorin e6; NBs, nanobubbles. 


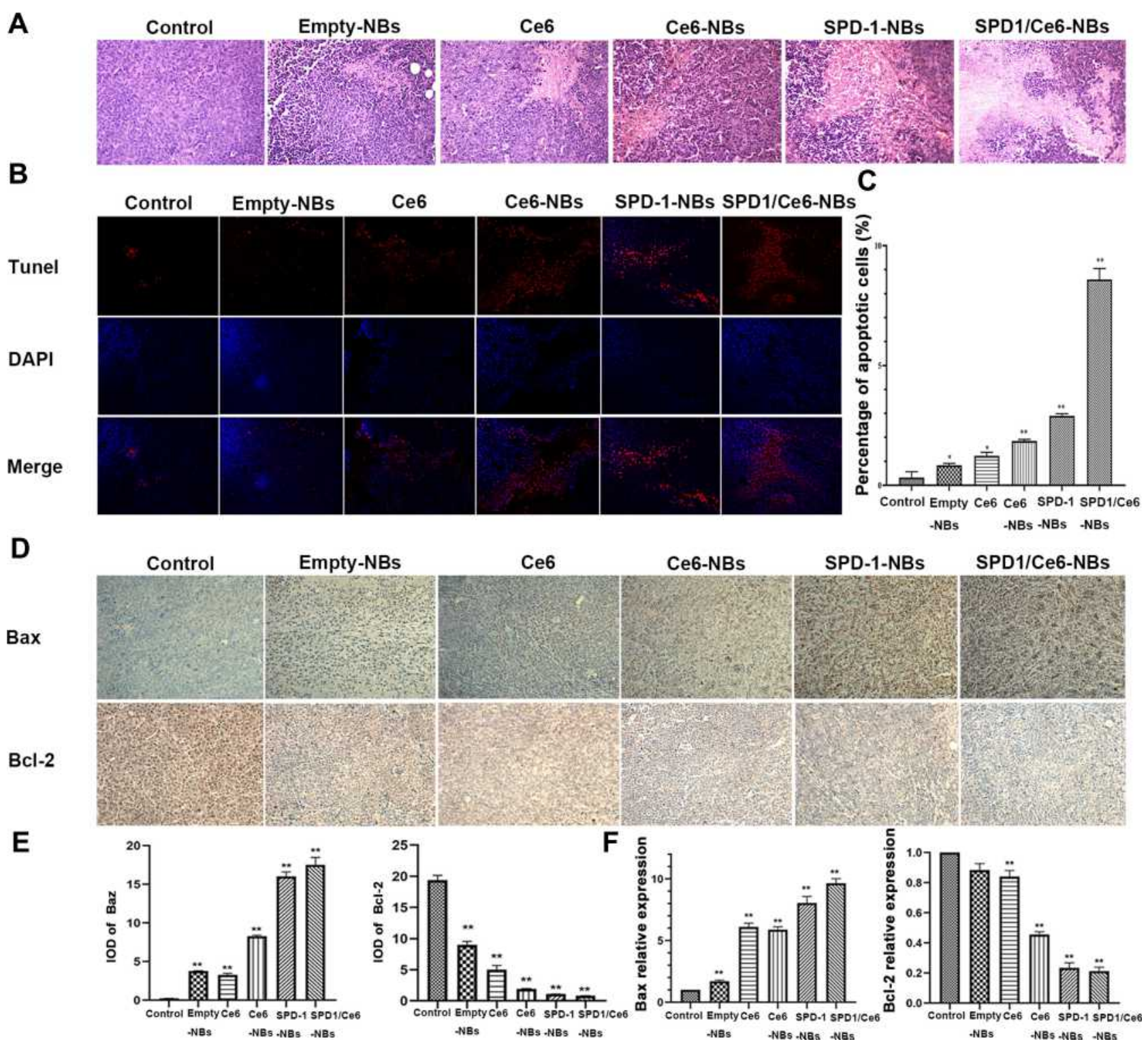

Figure 4 Analysis of apoptosis of tumor tissues, cells, protein and mRNA. (A) Histological examination by hematoxylin and eosin staining (magnification, x200). All treatments led to tissue apoptosis in varying degrees, with the SPD-I/Ce6-NBs group being the most obvious. (B) TUNEL images of tumor tissues (magnification, $x 200$ ). (C) Semi-quantification of the fluorescence intensity shown in panel (B). The trend of cell apoptosis was the same as that of tissues. (D) Immunochemistry staining images of Bax and $\mathrm{Bcl}-2$ (magnification, $\times 200$ ). (E) Protein expression of Bax and $\mathrm{Bcl}-2$. The expression of Bax protein in all the treatment groups was higher than that in the control groups, with the SPD-1/Ce6-NBs group being the most obvious. The opposite results were obtained for Bcl-2. (F) The mRNA expression of Bax and Bcl-2 followed the same trend as that of the protein. ( $* \mathrm{P}<0.05$, **P $<0.01$ versus the control group).

Abbreviations: Bax, B-cell lymphoma-2-associated X; Bcl-2, B-cell lymphoma-2; SPD-I, soluble programmed cell death I; Ce6, chlorin e6; NBs, nanobubbles; DAPI, 4', 6-diamidino-2-phenylindole; IOD, integral optical density.

which blocked the PD-1/PD-L1 signaling pathway by downregulating PD-L1 expression in tumor tissue. The results of immunohistochemistry staining and RT-qPCR revealed that protein expression (Figure $5 \mathrm{~A}$ and $\mathrm{C}$; both $P<0.01$ ) and the gene (Figure 5D) of PD-L1 was significantly lower in the sPD-1-NBs and sPD-1/Ce6-NBs groups compared with that of the control group. The opposite results were obtained for the gene of sPD-1 (Figure 5E). Thus, sPD-1 delivered by NBs could be used as an ICI. SDT elicits an immune response by inducing ICD, which can be verified by detecting the expression of calreticulin (CRT), a distinct marker of ICD cells. ${ }^{21}$ As shown in Figure $5 \mathrm{~B}$ and $\mathrm{F}$, the protein expression of CRT was significantly higher in the Ce6, Ce6-NB and sPD-1/Ce6-NBs groups compared with that of the control group $(P<0.01)$, indicating that Ce6assisted SDT can induce ICD and then trigger an immune response. 


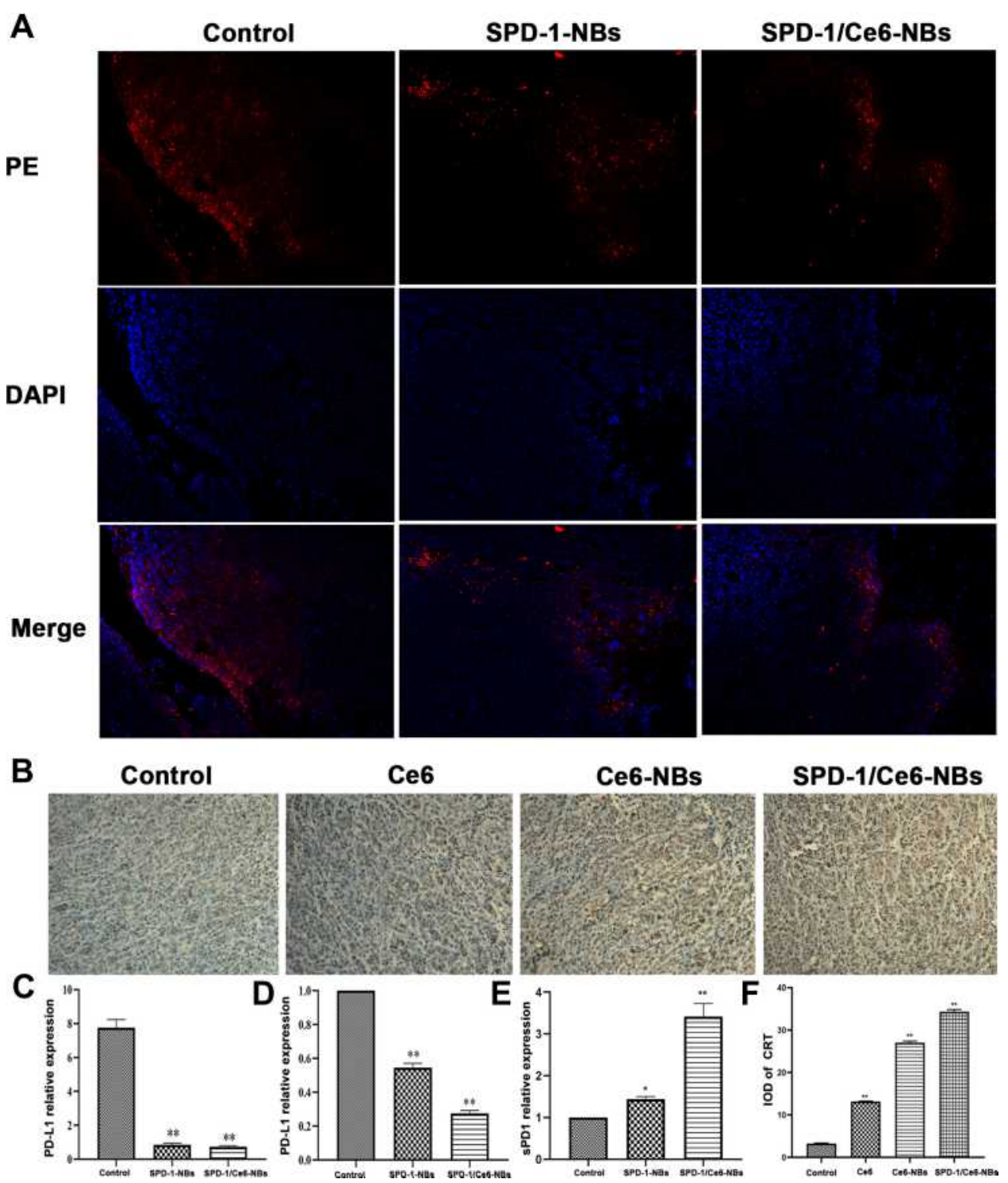

Figure 5 Antitumor mechanism of sPD-I-mediated immunotherapy and Ce6-assisted sonodynamic therapy. (A) Immunofluorescence images of PD-LI (magnification, x200). (B) Immunochemistry images of CRT (magnification, x200). (C) Semi-quantification of the fluorescence intensity shown in panel (A). Compared with the control group, the protein and mRNA expression of PD-LI shown in panels (C) and (D) was significantly lower in the SPD-I-NBs and SPD-I/Ce6-NBs groups. (E) The opposite results were obtained for the gene of sPD-I. The protein expression of CRT shown in panel (F) was significantly higher in the Ce6, Ce6-NB and SPD-I/Ce6-NBs groups. (*P $<0.05, * * \mathrm{P}<0.01$ versus the control group).

Abbreviations: PE, P-phycoerythrin; DAPI, 4',6-diamidino-2-phenylindole; SPD-I, soluble programmed cell death I; Ce6, chlorin e6; NBs, nanobubbles; PD-LI, programmed death-ligand I; IOD, integral optical density; CRT, calreticulin.

The present study explored whether ICIs combined with SDT resulted in a synergetic immunotherapy effect by evaluating the killing activities of CTLs and NKs, which are the main parts of cellular immunity and play an important role in immunotherapy. The CTLs and NKs of the Ce6-NBs, sPD-1-NBs and sPD-1/Ce6-NBs groups could effectively kill $\mathrm{H} 22$ cells. Compared with those of the control group, the killing activities of NKs and CTLs in the sPD-1/Ce6-NBs group were 2 and 20 -fold stronger, respectively (Figure 6A and $\mathrm{B}$; both $P<0.01$ ). Activation of cellular immunity could subsequently increase cytokine production and mature DCs. DCs maturity is demonstrated by the expression of CD80 and CD86, which are expressed in mature DCs. Thus, the mRNA expression levels of CD80 and CD86, and cytokines such as TNF- $\alpha$, IFN- $\gamma$ and IL-2, were examined. In all the treatment groups, the expression levels of CD80, CD86, TNF- $\alpha$, IFN- $\gamma$ and IL-2 were higher by 3 -5-fold compared with that of the control groups, with the differences being statistically significant, $P<0.01$ (Figure 6C). 

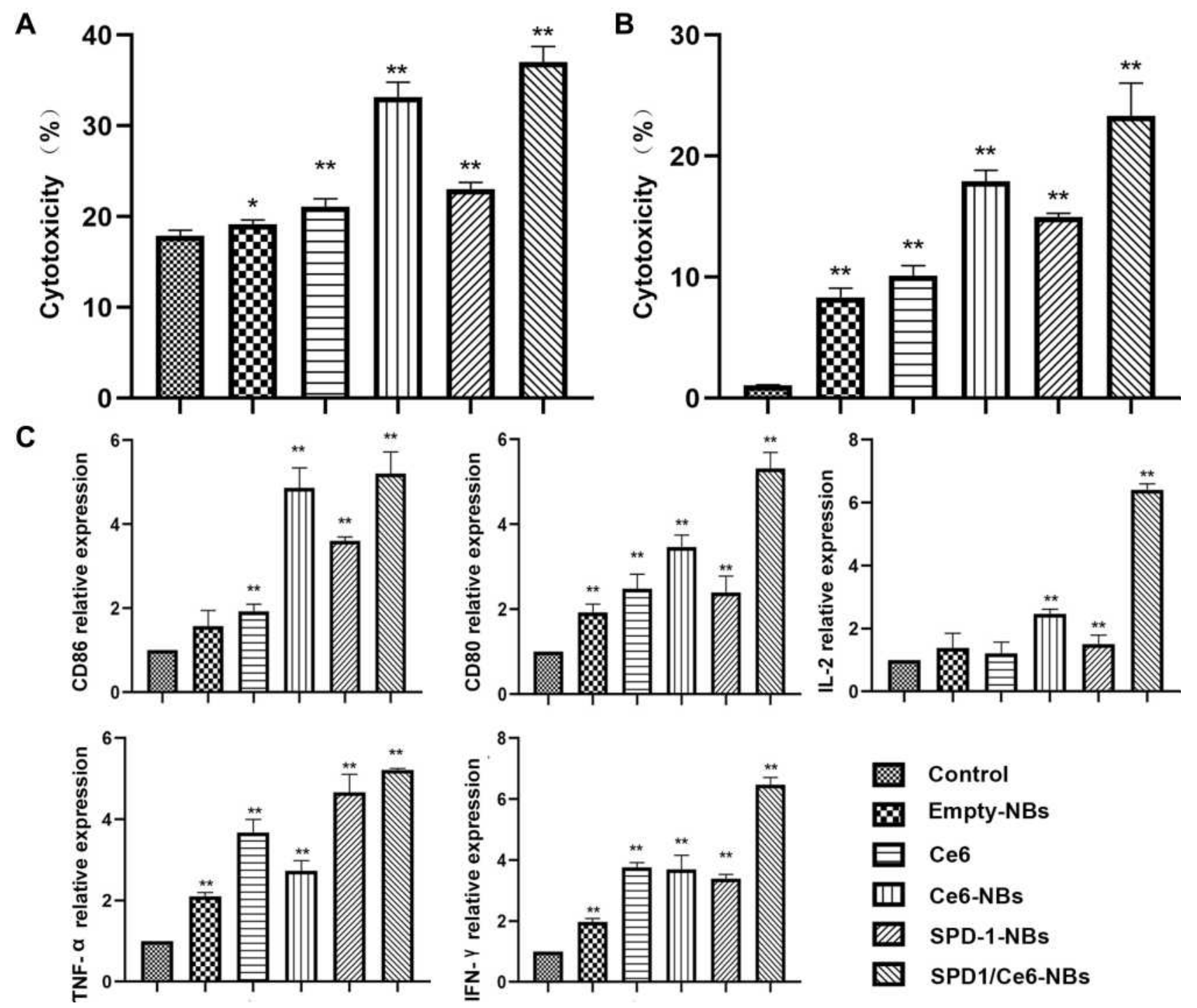

Figure 6 Immune activity enhanced by sPD-I/Ce6-NBs in vivo. The cytotoxic activity of natural killer splenic lymphocytes in panel (A) and cytotoxic T cell splenic lymphocytes in panel (B) was 2 and 20 -fold stronger, respectively, than that of the control group. (C) The mRNA expression levels of CD86, CD80, IFN- $\gamma$, TNF- $\alpha$ and IL-2 in the treatment groups were higher than those of the control group, which indicated that sPD-I/Ce6-NBs had good immune activity. $(* \mathrm{P}<0.05$, $* * \mathrm{P}<0.0 \mathrm{I}$ versus the control group).

Abbreviations: CD80, cluster of differentiation 80; TNF- $\alpha$, tumor necrosis factor alpha; IFN- $\gamma$, interferon gamma; IL-2, interleukin-2; PD-LI, programmed death-ligand I; SPD-I, soluble programmed cell death I; Ce6, chlorin e6; NBs, nanobubbles.

\section{Discussion}

Efficient and safe drug delivery is crucial for tumor therapy in vivo. The present study used NBs to deliver sPD-1 and $\mathrm{Ce} 6$ in a targeted manner. NBs had good dispersity and their $\mathrm{nm}$ size could lead to passive targeting due to the EPR effect. However, a shortcoming of our NBs is the absence of active targeting, which has been improved by other members in our group. ${ }^{22}$ They designed an actively targeted microbubbles conjugated with anti-PD-L1 mAb, which can target the PD-L1 site on the tumor cells. NBs in our study had both positive charge and lipid, which could result in a simultaneous loading of negatively charged sPD-1 plasmid and lipid-soluble Ce6, thus providing a necessary condition for synergetic immunotherapy. In our previous study, bubbles were used as non-viral systems for delivering paclitaxel and miR-34a gene in a targeted manner, effectively treating cervical cancer. ${ }^{23}$ FL imaging suggested that the NBs delivery system could increase the accumulated time and targeted concentration of $\mathrm{Ce} 6$ in the tumor, and further enhance the antitumor effects while avoiding systemic side effects. In addition, the tumor inhibition rate in the Ce6-NBs group was 
higher than that in the Ce6 group. Yue ${ }^{24}$ also used liposomes to acquire long-time retention and high accumulation of sonosensitizers in the tumor. Furthermore, the tumor inhibition rate in the empty NBs group indicated that NBs, combined with UTND, were targeted anticancer systems and played an effective role in noninvasive tumor therapy. $\mathrm{Gao}^{25}$ used phase-change nanodroplets to deliver drugs and for diagnosing tumors. Due to UTND, nanodroplets can achieve a high drug uptake and imaging contrast. Regarding side effects, lipid materials were used to fabricate NBs, which is widely used in the clinic. According to the mice weight curve and liver index results, the NBs in present study had a good biocompatibility and showed no toxicity towards mice.

The sPD-1 plasmid delivered by NBs could stably upregulate sPD-1 expression in tumors, which downregulated PD-L1 expression, thus blocking PD-L/PD-1 interactions. This resulted in CD4+ and CD8+ T lymphocytes activation and proliferation by activating $\mathrm{T}$ cell receptors, which then modulated tumor immunosuppressive environments. This is the antitumor mechanism of sPD-1-assisted immunotherapy. In our previous study, sPD1 plasmid was used to increase the secretion of related cytokines, inducing apoptosis and enhancing antitumor immunity in a cervical cancer U14 subcutaneous xenograft mouse model. ${ }^{26}$

To achieve a synergistic effect on tumor treatment, checkpoint blockade has been combined with multiple therapeutic modalities, including radiation, ${ }^{27}$ chemotherapy, ${ }^{28}$ anti-angiogenesis, ${ }^{29}$ and $\mathrm{PDT}^{30}$ However, all these modalities have various disadvantages, including radiation damage, toxicity and poor tissue-penetrating, which hampers their broad clinical application. The present study used Ce6-assisted SDT to further strengthen its antitumor activity, ${ }^{31}$ which was demonstrated in the results of tumor inhibition rate, histopathological changes, cell apoptosis, and expression of apoptosis-related genes and proteins. SDT can generate ROS, such as singlet oxygen, which not only can result in extensive tumor cell death, but also trigger immune responses by generating ICD. ${ }^{32}$ ICD translocates CRT to the cell surface, which is an "eat signal" to activate and maturate DCs and macrophages, ${ }^{21}$ and then increases the number of effector $\mathrm{T}$ cells in the tumor microenvironment. ${ }^{33}$ The observed upregulation of CRT expression demonstrated this process. Ce6 was used as a sonosensitizer, which had a preferential localization in tumors, while low accumulation and rapid clearance in normal tissues, thus enhancing the suppressing tumor effect without evident side effects. In a previous study, $\mathrm{Ce} 6, \mathrm{Mn}^{2+}$ and human serum albumin were mixed to combine nanoassemblies. $\mathrm{Ce} 6$ and $\mathrm{Mn}^{2+}$ can be used for tumor-targeting fluorescence and magnetic resonance imaging in vivo, thus achieving imaging-guided SDT therapy. ${ }^{34}$ The lack of imaging guided therapy is a shortcoming of our study, however, NBs can be used as novel carriers and to enhance ultrasound imaging, which can locate tumors and evaluate the therapeutic effect during treatment. Thus, our future studies will employ NBs and Ce6 to achieve integration of diagnosis and treatment.

The present study demonstrated that sPD-1/Ce6-NBs could be used for SPD-1 checkpoint blockade therapy combined with Ce6-assisted SDT to enhance immune responses, inhibiting tumor growth significantly. sPD-1/Ce6-NBs could activate immune effector cells, such as NKs and CTLs. T cells could be differentiated into helper $\mathrm{T}$ cells (Th1 and Th2), thus increasing the secretion of IFN- $\gamma$, TNF$\alpha$ and IL-2, which are involved in the cell-mediated immune response. ${ }^{35}$ Upregulation of the expression of IFN- $\gamma$ and TNF- $\alpha$ triggered the maturation of DCs, which was demonstrated by the upregulated expression of CD80 and CD86, which are expressed on the surface of mature DCs. Mature DCs could further activate NKs and CTLs. ${ }^{36,37}$ The increase in Interleukin-2 (IL-2) could induce various cytokines cascade reactions, which maintained $\mathrm{CD} 4+$ and $\mathrm{CD} 8+\mathrm{T}$ cell proliferation. Furthermore, $\mathrm{Yue}^{24}$ reported that the combination of anti-PD-L1 checkpoint blockade-enabled immunotherapy and nanosonosensitiser-augmented SDT can efficiently prevent tumor growth and metastasis in mice. Although the present study only demonstrated that NBs could inhibit the primary tumor directly, it can be speculated that, theoretically, sPD-1/Ce6-NBs could induce a long-term immune memory function and systemic anticancer immunity, which could prevent tumor reoccurrence and inhibit distant metastasis.

\section{Conclusion}

In conclusion, in the present study, a type of multifunctional nanoparticle was rationally designed. SPD-1/ Ce6-NBs not only achieved targeted delivery and local release of Ce6 as well as sPD-1, but they also induced UTND, thus enhancing their antitumor effect. sPD-1 checkpoint blockade therapy, further promoted by Ce6induced SDT, increased the production of immune factors and the killing effect of NKs as well as CTLs, which resulted in effective synergetic immunotherapy for HCC. 
The present study provides a new insight for the development of a widely established cancer therapy with significant potential in clinical applications.

\section{Disclosure}

The authors report no conflicts of interest in this work.

\section{References}

1. Bray F, Ferlay J, Soerjomataram I, et al. Global cancer statistics 2018: GLOBOCAN estimates of incidence and mortality worldwide for 36 cancers in 185 countries. CA Cancer J Clin. 2018;68 (6):394-424. doi:10.3322/caac.21492

2. Chen Q, Chen M, Liu Z. Local biomaterials-assisted cancer immunotherapy to trigger systemic antitumor responses. Chem Soc Rev 2019;48(22):5506-5526. doi:10.1039/c9cs00271e

3. Li ZW, Li B, Peng D, et al. Expression and clinical significance of PD1 in hepatocellular carcinoma tissues detected by a novel mouse anti-human PD1 monoclonal antibody. Int $J$ Oncol. 2018;52 (6):2079-2092. doi:10.3892/ijo.2018.4358

4. Feng Z, Rong P, Wang W. Meta-analysis of the efficacy and safety of PD-1/PD-L1 inhibitors administered alone or in combination with anti-VEGF agents in advanced hepatocellular carcinoma. Gut. 2020;69(10):1904-1906. doi:10.1136/gutjnl-2019-320116

5. Cheng AL, Hsu C, Chan SL, Choo SP, Kudo M. Challenges of combination therapy with immune checkpoint inhibitors for hepatocellular carcinoma. $J$ Hepatol. 2020;72(2):307-319. doi:10.1016/j. jhep.2019.09.025

6. Chen B, Zheng R, Liu D, Li B, Lin J, Zhang W. The tumor affinity of chlorin $\mathrm{e} 6$ and its sonodynamic effects on non-small cell lung cancer. Ultrason Sonochem. 2013;20(2):667-673. doi:10.1016/j. ultsonch.2012.09.008

7. Wang P, Li C, Wang X, et al. Anti-metastatic and pro-apoptotic effects elicited by combination photodynamic therapy with sonodynamic therapy on breast cancer both in vitro and in vivo. Ultrason Sonochem. 2015;23:116-127. doi:10.1016/j.ultsonch.2014.10.027

8. Shi H, Liu Q, Qin X, Wang P, Wang X. Pharmacokinetic study of a novel sonosensitizer chlorin-e6 and its sonodynamic anti-cancer activity in hepatoma-22 tumor-bearing mice. Biopharm Drug Dispos. 2011;32(6):319-332. doi:10.1002/bdd.761

9. Feng Q, Li Y, Yang X, et al. Hypoxia-specific therapeutic agents delivery nanotheranostics A sequential strategy for ultrasound mediated on-demand tritherapies and imaging of cancer. $J$ Control Release. 2018;275:192-200. doi:10.1016/j.jconrel.2018.02.011

10. Schieber M, Chandel NS. ROS function in redox signaling and oxidative stress. Curr Biol. 2014;24(10):R453-462. doi:10.1016/j. cub.2014.03.034

11. Son J, Yi G, Kwak MH, et al. Gelatin-chlorin e6 conjugate for in vivo photodynamic therapy. $J$ Nanobiotechnology. 2019;17(1):50. doi:10.1186/s12951-019-0475-1

12. Suzuki R, Maruyama K. Development of Ultrasound theranostics for cancer. Yakugaku Zasshi. 2018;138(7):919-922. doi:10.1248/ yakushi.17-00220-3

13. Kosareva A, Abou-Elkacem L, Chowdhury S, Lindner JR, Kaufmann BA. Seeing the invisible-ultrasound molecular imaging. Ultrasound Med Biol. 2020;46(3):479-497. doi:10.1016/j. ultrasmedbio.2019.11.007

14. de Leon A, Perera R, Nittayacharn P, Cooley M, Jung O, Exner AA. Ultrasound contrast agents and delivery systems in cancer detection and therapy. Adv Cancer Res. 2018;139:57-84. doi:10.1016/bs. acr.2018.04.002
15. Akbaba H, Erel-Akbaba G, Kotmakçı M, Başpınar Y. Enhanced cellular uptake and gene silencing activity of survivin-siRNA via ultrasound-mediated nanobubbles in lung cancer cells. Pharm Res. 2020;37(8):165. doi:10.1007/s11095-020-02885-x

16. Wang X, Wang Y, Wang P, Cheng X, Liu Q. Sonodynamically induced anti-tumor effect with protoporphyrin IX on hepatoma-22 solid tumor. Ultrasonics. 2011;51(5):539-546. doi:10.1016/j. ultras.2010.12.001

17. Sun $\mathrm{S}, \mathrm{Xu} \mathrm{Y}, \mathrm{Fu}$ P, et al. Ultrasound-targeted photodynamic and gene dual therapy for effectively inhibiting triple negative breast cancer by cationic porphyrin lipid microbubbles loaded with HIF 1alpha-siRNA. Nanoscale. 2018;10(42):19945-19956. doi:10.1039/c8nr03074j

18. Izadifar Z, Babyn P, Chapman D. Mechanical and biological effects of ultrasound: a review of present knowledge. Ultrasound Med Biol. 2017;43(6):1085-1104. doi:10.1016/j.ultrasmedbio.2017.01.023

19. Zhou Y, Gu HT, Xu Y, et al. Targeted antiangiogenesis gene therapy using targeted cationic microbubbles conjugated with CD105 antibody compared with untargeted cationic and neutral microbubbles. Theranostics. 2015;5(4):399-417. doi:10.7150/thno.10351

20. Mura P, Maestrelli F, González-Rodríguez ML, Michelacci I, Ghelardini C, Rabasco AM. Development, characterization and in vivo evaluation of benzocaine-loaded liposomes. Eur J Pharm Biopharm. 2007;67(1):86-95. doi:10.1016/j.ejpb.2007.01.020

21. Hwang HS, Shin H, Han J, Na K. Combination of photodynamic therapy (PDT) and anti-tumor immunity in cancer therapy. $J$ Pharm Investig. 2018;48(2):143-151. doi:10.1007/s40005-017-0377-x

22. Ma Y, Han JX, Jiang JJ, et al. Ultrasound targeting of microbubble-bound anti PD-L1 $\mathrm{mAb}$ to enhance anti-tumor effect of cisplatin in cervical cancer xenografts treatment. Life Sci. 2020;262:118565. doi:10.1016/j.lfs.2020.118565

23. Yu J, Zhao Y, Liu C, et al. Synergistic anti-tumor effect of paclitaxel and miR-34a combined with ultrasound microbubbles on cervical cancer in vivo and in vitro. Clin Transl Oncol. 2020;22(1):60-69. doi:10.1007/s12094-019-02131-w

24. Yue W, Chen L, Yu L, et al. Checkpoint blockade and nanosonosensitizer-augmented noninvasive sonodynamic therapy combination reduces tumour growth and metastases in mice. Nat Commun. 2019;10(1):2025. doi:10.1038/s41467-019-09760-3

25. Gao J, Yu B, Li C, et al. Ultrasound triggered phase-change nanodroplets for doxorubicin prodrug delivery and ultrasound diagnosis: an in vitro study. Colloids Surf B Biointerfaces. 2019;174:416-425. doi:10.1016/j.colsurfb.2018.11.046

26. Qin YE, Tang WF, Xu Y, Wan FR, Chen AH. Ultrasound-mediated co-delivery of miR-34a and SPD-1 complexed with microbubbles for synergistic cancer therapy. Cancer Manag Res. 2020;12:2459-2469. doi:10.2147/CMAR.S238643

27. Sharabi AB, Lim M, Deweese TL, Drake CG. Radiation and checkpoint blockade immunotherapy: radiosensitisation and potential mechanisms of synergy. Lancet Oncol. 2015;16(13):e498-e509. doi:10.1016/S1470-2045(15)00007-8

28. Grasselly C, Denis M, Bourguignon A, Talhi N, Dumontet C. The antitumor activity of combinations of cytotoxic chemotherapy and immune checkpoint inhibitors is model-dependent. Front Immunol. 2018;9:210. doi:10.3389/fimmu.2018.02100

29. Song W, Kuang J, Li CX, et al. Enhanced immunotherapy based on photodynamic therapy for both primary and lung metastasis tumor eradication. ACS Nano. 2018;12(2):1978-1989. doi:10.1021/ acsnano.7b09112

30. Xu J, Yu S, Wang X, et al. High affinity of chlorin e6 to immunoglobulin $\mathrm{g}$ for intraoperative fluorescence image-guided cancer photodynamic and checkpoint blockade therapy. ACS Nano. 2019;13(9):10242-10260. doi:10.1021/acsnano.9b03466

31. Zhang Q, Bao C, Cai X, et al. Sonodynamic therapy-assisted immunotherapy: a novel modality for cancer treatment. Cancer Sci. 2018;109(5):1330-1345. doi:10.1111/cas.13578 
32. Zhang N, Tan Y, Yan L, et al. Modulation of tumor hypoxia by $\mathrm{pH}$-responsive liposomes to inhibit mitochondrial respiration for enhancing sonodynamic therapy. Int $J$ Nanomedicine. 2020;15:5687-5700. doi:10.2147/IJN.S256038

33. Panaretakis T, Joza N, Modjtahedi N, et al. The co-translocation of ERp57 and calreticulin determines the immunogenicity of cell death. Cell Death Differ. 2008;15(9):1499-1509. doi:10.1038/cdd.2008.67

34. Wan Q, Zou C, $\mathrm{Hu} \mathrm{D}$, et al. Imaging-guided focused ultrasound-induced thermal and sonodynamic effects of nanosonosensitizers for synergistic enhancement of glioblastoma therapy. Biomater Sci. 2019;7(7):3007-3015. doi:10.1039/c9bm00292h
35. Xu-Monette ZY, Mingzhi Z, Jianyong L. PD-1/PD-L1 blockade: have we found the key to unleash the antitumor immune response? Front Immunol. 2017;8:1597. doi:10.3389/fimmu.2017.01597

36. Wathelet N, Moser M. Role of dendritic cells in the regulation of antitumor immunity. Oncoimmunology. 2013;2(4):e23973. doi:10.4161/onci.23973

37. Hu W, Jain A, Gao Y, et al. Differential outcome of TRIF-mediated signaling in TLR4 and TLR3 induced DC maturation. Proc Natl Acad Sci $U \quad S \quad$ A. 2015;112(45):13994-13999. doi:10.1073/ pnas. 1510760112

\section{Publish your work in this journal}

The International Journal of Nanomedicine is an international, peerreviewed journal focusing on the application of nanotechnology in diagnostics, therapeutics, and drug delivery systems throughout the biomedical field. This journal is indexed on PubMed Central, MedLine, CAS, SciSearch ${ }^{\mathbb{R}}$, Current Contents ${ }^{\mathbb{R}} /$ Clinical Medicine, $^{-}$
Journal Citation Reports/Science Edition, EMBase, Scopus and the Elsevier Bibliographic databases. The manuscript management system is completely online and includes a very quick and fair peer-review system, which is all easy to use. Visit http://www.dovepress.com/ testimonials.php to read real quotes from published authors. 